\title{
Curcumin induces p53-independent necrosis in H1299 cells via a mitochondria-associated pathway
}

\author{
FEIE LI ${ }^{1}$, XI CHEN ${ }^{2}$, BING XU ${ }^{2}$ and HUA ZHOU ${ }^{3}$ \\ ${ }^{1}$ Department of Pharmacy, Tongji Hospital of Tongji Medical College; ${ }^{2}$ School of Public Health, \\ Tongji Medical College; ${ }^{3}$ Department of Pediatrics, Tongji Hospital of Tongji Medical College, \\ Huazhong University of Science and Technology, Wuhan, Hubei 430030, P.R. China
}

Received November 11, 2014; Accepted September 9, 2015

DOI: $10.3892 / \mathrm{mmr} .2015 .4395$

\begin{abstract}
Curcumin has been shown to have various therapeutic and/or adjuvant therapeutic effects on human cancers, as it inhibits cancer cell proliferation and induces apoptosis through p53-dependent molecular pathways. However, numerous cancer cell types bear a mutant p53 gene, and whether curcumin has any therapeutic effects on p53-deficient/mutant cancer cells has remained elusive. The present study sought to determine whether curcumin exerts any anti-proliferative and cytotoxic effects on the p53-deficient H1299 human lung cancer cell line via a p53-independent mechanism. An MTT assay and flow cytometric analysis indicated that curcumin significantly decreased cell proliferation and induced necrotic cell death. Western blot analysis of the cytosolic and mitochondrial fractions of H1299 cells as well as a fluorometric caspase assay indicated that curcumin-induced necrosis was mitochondria- and caspase-dependent, and resulted in cytochrome $c$ release. Of note, this necrotic cell death was reduced following inhibition of B-cell lymphoma-2 (Bcl-2)-associated $\mathrm{X}$ protein $(\mathrm{Bax})$ or $\mathrm{Bcl}-2$ homologous antagonist killer (Bak) as well as overexpression of Bcl-2. In conclusion, the present study suggested that curcumin-induced necrotic cell death was mediated via a p53-independent molecular pathway, which was associated with Bax and Bak translocation, caspase activation and cytochrome $c$ release.
\end{abstract}

Correspondence to: Professor Hua Zhou, Department of Pediatrics, Tongji Hospital of Tongji Medical College, Huazhong University of Science and Technology, 1095 Jiefang Road, Wuhan, Hubei 430030, P.R. China

E-mail: huazhouwh2014@163.com

Abbreviations: DMSO, dimethyl sulfoxide; Bcl-2, B-cell lymphoma 2; BAX, Bcl-2-associated X protein; Bak, Bcl-2 homologous antagonist killer

Key words: curcumin, H1299 cells, B-cell lymphoma-2-associated $\mathrm{X}$ protein, mitochodria, necrosis

\section{Introduction}

Curcumin, a polyphenol isolated from the rhizomes of the turmeric plant (Curcuma longa), is known to exhibit potent anti-neoplastic activity against a variety of human cancer types, including pancreatic, colon, ovarian and breast cancer, by modulating multiple signaling pathways (1-3). Curcumin has been reported to activate p53 signaling pathways, which upregulates the transcription of downstream genes, including B-cell lymphoma-2 (Bcl-2)-associated X protein (Bax) and Bcl-2 homologous antagonist killer (Bak), and downregulates pro-survival genes, including $\mathrm{Bcl}-2$ and cyclin $\mathrm{D} 1$, to induce apoptosis in a variety of cancer cells $(4,5)$. However, the tumor suppressor gene p53 is mutated or deficient in a large percentage of human malignancies, and whether or not curcumin has anti-cancer effects on these p53 deficient/mutant tumors has remained elusive.

Programmed cell death (PCD) has an important biological function in development and homeostasis in mammals. A common type of PCD is apoptosis, which may occur in multicellular organisms in response to endogenous or exogenous cellular injuries and molecular stimuli, including death ligands such as tumor necrosis factor-alpha (TNF- $\alpha$ ), Fas ligand/CD95/apolipoprotein 1 and TNF-related apoptosis-inducing ligand, as well as DNA damaging agents, including benzo[a]pyrene, etoposide and 5-fluorouracil (6). In addition, induction of apoptosis is a key mechanism of action of cytotoxic anti-cancer drugs $(7,8)$. Cell necrosis is an alternative form of PCD, which may, in contrast to apoptosis, proceed without activation of caspase family members. Programmed cell necrosis may participate in vital processes when caspase members are inhibited by pharmacological or genetic means, i.e. when interdigital webbing is removed during embryogenesis in apoptotic protease activating factor 1 (Apaf1)-deficient embryos $(9,10)$. Similar to apoptosis, programmed cell necrosis can be activated by several key mediators, which have been identified through a genome-wide RNA interference screening; these include receptor-interacting protein kinase-1 (RIP1), RIP3, mitochondrial phosphoglycerate mutase family member 5 and mixed lineage kinase domain-like proteins (11). Cells may undergo programmed necrosis as an alternative mode of cell death when the caspase machinery fails or is inactivated (e.g. after viral infection or in apoptosis-resistant 
tumor cells) (12,13). Variable doses of stress may induce certain molecular events which are involved in apoptosis as well as necrosis signaling; therefore, cross-talk between the two cell death mechanisms exists $(14,15)$. This suggests that necrosis is an intrinsic pathway, which may be equally important to apoptosis; however, the underlying molecular mechanisms of programmed necrosis have remained to be fully elucidated.

The tumor suppressor p53 has crucial roles in apoptosis, genomic stability and inhibition of angiogenesis (16). Upon activation, p53 can halt cell cycle progression by inhibiting G0/G1 phase transition, allowing for DNA repair factors to fix the DNA damage. If the extent of DNA damage proves to be irreparable, p53 initiates apoptosis through transcriptional activation of a series of target proteins, including MDM2 and the pro-apoptotic proteins Bax and Bak (17); or through transcriptional inhibition of pro-survival protein Bcl-xl and Bcl-2 (18). Previous studies have also demonstrated important roles for p53 in curcumin-induced apoptosis. For instance, curcumin can induce human HT-29 colon adenocarcinoma cell apoptosis by activating p53 and regulating apoptosis-associated protein expression (19). In another study, curcumin treatment was shown to improve the general health of patients with colorectal cancer via increasing p53 expression in tumor cells and to consequently accelerate tumor cell apoptosis (4). Loss of p53 function and the resulting evasion of apoptosis is the most common event during tumorigenesis in diverse types of human cancer, and the efficacy of apoptosis-inducing anti-cancer drugs is therefore decreased (20). To date, little is known regarding the efficacy of curcumin on p53-mutant/deficient cancer cells as well as the underlying mechanisms.

The present study sought to explore the p53-independent mechanisms of action of curcumin in lung cancer cells. Using a previous study as a guideline (21), curcumin at the concentration of $10 \mu \mathrm{M}$ was selected for treating $\mathrm{p} 53$-proficient A549 cells and p53-deficient H1299 cells. The effects of curcumin on the proliferation, cytosolic and mitochondrial expression as well as homo-oligomerization of Bax and Bak, cytochrome $c$ release and cell death in A549 and H1299 cells was assessed using an MTT assay, western blot analysis and flow cytometry. Furthermore, the caspase-dependency of the curcumin-induced cytotoxicity was tested using a fluorometric assay in the presence of caspase inhibitors. In addition, the effects of small interfering (si)RNA-mediated Bax and Bak knockdown as well as Bcl-2 overexpression on the necrotic death of H1299 cells were assessed. The present study suggested that curcumin induces necrosis in p53-deficient H1299 cells through mitochondrial-associated molecular pathways.

\section{Materials and methods}

Drugs. Curcumin (Sigma-Aldrich, St. Louis, MO, USA) was dissolved in dimethyl sulfoxide (DMSO; Sigma-Aldrich) at $10 \mathrm{mM}$ as a stock solution. The dilutions of all of the reagents were freshly prepared prior to each experiment.

Cell lines. The p53-proficient lung cancer cell line A549 and the p53-deficient cell line H1299 were supplied by the Cell Bank of Type Culture Collection of the Chinese Academy of Science (Shanghai, China). A549 and H1299 cells were grown in Dulbecco's modified Eagle's medium (DMEM; Gibco, Thermo Fisher Scientific, Waltham, MA, USA) and in RPMI 1640 medium (Gibco) supplemented with 10\% (v/v) fetal bovine serum (FBS; Gibco). The two cell lines were incubated at $37^{\circ} \mathrm{C}$ in a humidified atmosphere containing $5 \% \mathrm{CO}_{2}$.

Reagents and antibodies. Rabbit polyclonal anti-p53 (cat. no. BS1278), rabbit polyclonal anti-BAX (cat. no. BS1725), rabbit polyclonal anti-BAK (cat. no. BS6477) and rabbit polyclonal MDM2 (cat. no. BS6818) primary antibodies were purchased from Bioworld Technology (St. Louis Park, MA, USA); primary antibody against Bcl-2 (cat. no. sc-492) was obtained from Santa Cruz Biotechnology (Dallas, TX, USA); rabbit polyclonal anti-cytochrome $c$ (cat. no. BA0781) was supplied by Boster Bio-Engineering (Wuhan, China); rabbit monoclonal anti-cyclooxygenase (Cox)IV (cat. no. 18-7379) was purchased from Molecular Probes (Thermo Fisher Scientific); and all peroxidase-conjugated goat anti-rabbit secondary antibodies (cat. no. 00008-2) were obtained from Bioworld Technology. Chemical cross-linking agent bismaleimidohexane (BMH), was supplied by Pierce Biotechnology, Inc. (Rockford, IL,USA). The carbobenzoxy-Val-Ala-Asp fluoromethyl ketone (Z-VAD-FMK), Asp-Glu-Val-Asp (DEVD)-7-amino-4-trifluoromethyl coumarin (AFC) were obtained from Selleck Chemicals (Houston, TX, USA); and propidium iodide (PI) and Annexin V fluorescein isothiocyanate (FITC) double staining apoptosis detection kit was a product of Biotech (Hangzhou, China). The mitochondria/cytosol protein isolation kit was obtained from KeyGen (Nanjing, China) and Lipofectamine 2000 transfection reagent was from Invitrogen (Thermo Fisher Scientific).

Growth and cell proliferation analysis. A549 and H1299 cells were seeded in 96-well microplates and treated with $10 \mu \mathrm{M}$ curcumin. The controls were treated with an equal volume of the vehicle (DMSO; final concentration, $\leq 0.1 \%$ ). Following incubation, the supernatant in each well was replaced with $90 \mu \mathrm{l} \mathrm{FBS}$-free medium and $10 \mu \mathrm{l}$ MTT solution (final concentration, $5 \mathrm{mg} / \mathrm{ml}$ ). The plates were then further incubated at $37^{\circ} \mathrm{C}$ for $4 \mathrm{~h}$. Subsequently, the supernatant in each well was carefully aspirated and $150 \mu \mathrm{l}$ DMSO was added to dissolve the MTT formazan. Following 15 min of incubation, the absorbance of each well was read using a microplate reader (model 680; Bio-Rad Laboratories, Inc., Hercules, CA, USA) at $570 \mathrm{~nm}$ wavelength. Three independent experiments using six parallel wells were performed.

Flow cytometric analysis. H1299 cells were seeded into six-well plates at density of $5 \times 10^{5}$ cells/well. The cells were treated with $10 \mu \mathrm{M}$ curcumin. After incubation for $0,8,16$ and 24 h, H1299 cells were collected and incubated with $5 \mu \mathrm{l}$ Annexin V-FITC and $10 \mu \mathrm{l}$ PI per $100 \mu \mathrm{l}$ binding buffer in the dark for $20 \mathrm{~min}$ at room temperature. Finally, cell death mode analysis was performed using a FACSort flow cytometer (BD Biosciences, Franklin Lakes, NJ, USA). For each group, 10,000 events were collected and analyzed using Cell Quest software.

Measurement of caspase activity. H1299 cells were plated in six-well plates at a density of $8 \times 10^{5}$ cells/well and subse- 
quently treated with $10 \mu \mathrm{M}$ curcumin. After incubation for 0 , 8,16 and $24 \mathrm{~h}$, the cells were collected and extracted with $1 \%$ Triton X-100 (Sigma-Aldrich). A total of $25 \mu \mathrm{g}$ whole-cell lysate was subjected to an enzymatic reaction in the presence of $50 \mathrm{M} \mathrm{DEVD}-\mathrm{AFC}$ at $37^{\circ} \mathrm{C}$ for $1 \mathrm{~h}$. The fluorescent emission (excitation at $360 \mathrm{~nm}$ and emission at $530 \mathrm{~nm}$ ) was measured. In addition, a standard curve was plotted for each measurement using free AFC. Using the standard curve, the fluorescence intensity of each enzymatic reaction was determined. The caspase activity was expressed in $\mathrm{pmol} / \mathrm{min} / \mathrm{mg}$ protein.

Bax/Bak siRNA transfection. To inhibit the expression of Bax or Bak in H1299 cells, gene silencing was performed using the Lipofectamine 2000 transfection reagent according to the manufacturer's instructions. The target siRNA and non-specific control siRNA sequences were as follows: Bak (beginning at nt 310), 5'-P-UGCCUACGAACUCUUCACCdTdT-3' (sense) and 5'-P-GGUGAAGAGUUCGUAGGCAdTdT-3' (anti-sense); Bax (beginning at nt 217), 5'-P-UAUGGA GCUGCAGAGGAUGdTdT-3' (sense) and 5'-P-CAUCCUCUG CAGCUCCAUAdTdT-3' (anti-sense); non-specific control siRNA, 5'-NNATTGTATGCGATCGCAGAC-3'. All of the above siRNA sequences were designed and synthesized by GenePharma (Shanghai, China). For transfection, H1299 cells were seeded into six-well plates at $3 \times 10^{5}$ cells per well. After incubation for $24 \mathrm{~h}$, the cells were transfected with the specific Bax or Bak siRNA sequence using Lipfectamine 2000 transfection agent following the manufacturer's instructions. After $36 \mathrm{~h}$ of transfection, the cells were harvested for further study.

Western blot analysis. Western blotting was performed to determine the expression levels of apoptotic signaling molecules and their associated mitochondrial molecules in H1299 cells. Briefly, after incubation with $10 \mu \mathrm{M}$ curcumin, $1 \times 10^{7} \mathrm{H} 1299$ cells were harvested for protein extraction. Mitochondrial and mitochondria-free proteins were separated according to the instruction manual of the kit (KeyGen). The whole-cell lysate was extracted using radioimmunoprecipitation assay buffer $(1 \mathrm{M}$ Tris- $\mathrm{HCl}, 5 \mathrm{M} \mathrm{NaCl}, 1 \%$ Nonidet P-40, $1 \%$ sodium deoxycholate, $0.05 \%$ SDS and $1 \mathrm{mM}$ phenylmethyl sulfonyl fluoride). Western blot analysis was then performed as previously described using SDS buffer $(25 \mathrm{mM}$ Tris, $192 \mathrm{mM}$ glycine, $0.1 \%$ SDS) (22). The blots were subsequently incubated with the specific primary antibodies (1:400 dilution for all) at $4^{\circ} \mathrm{C}$ overnight, and with the corresponding IR dye-conjugated secondary antibodies (1:5,000 dilution for all) at room temperature for $1 \mathrm{~h}$. Membranes were visualized using the Odyssey Infrared Imaging System and Odyssey v1.2 (LI-COR Biosciences, Lincoln, NE, USA). The relative densities of the protein bands were analyzed using Quantity One software (Bio-Rad Laboratories, Inc.).

Bax/Bak homo-oligomerization. The membrane-permeable cross-linking reagent $\mathrm{BMH}$ was used to analyze Bax/Bak oligomerization. Briefly, after incubation with indicated concentrations of curcumin, $1 \times 10^{7}$ of H1299 cells were collected for protein extraction. The mitochondrial-membrane fractions were separated by ultracentrifugation at $100,000 \mathrm{x} \mathrm{g}$ for $60 \mathrm{~min}$ at $2-8^{\circ} \mathrm{C}$ and re-suspended in $250 \mu \mathrm{l}$ phosphate-buffered saline (PBS). The collected mitochondrial-membrane fraction was then incubated with $0.2 \mathrm{mM}$ BMH in PBS for $1 \mathrm{~h}$ at room temperature with agitation at $12 \mathrm{x} \mathrm{g} / \mathrm{min}$. Finally, the above mixtures were dissolved by 4 X SDS buffer, subjected to $12 \%$ SDS-PAGE and analyzed by immunoblot analysis using the corresponding primary antibodies.

Bcl-2 overexpression in H1299 cells. To establish Bcl-2-overexpressing H1299 cells, a Bcl-2 expression plasmid, pcDNA3-Bcl-2, was constructed (23). For transfection of the plasmid, H1299 cells were plated into six-well plates at $3 \times 10^{5}$ cells per well. The transfection procedure was performed as previously described with minimal revision (22).

Statistical analysis. Values are expressed as the mean \pm standard deviation. Data were analyzed using a t-test. All statistical analyses were performed using SPSS, version 13.0 for Windows (SPSS Inc., Chicago, IL, USA). P $<0.05$ was considered to indicate a statistically significant difference between values.

\section{Results}

Curcumin inhibits A549 and H1299 cell proliferation. The effects of $10 \mu \mathrm{M}$ curcumin on A549 and H1299 cell viability were assessed using an MTT assay at 8, 16 and $24 \mathrm{~h}$ post-treatment. The deficiency of p53 in H1299 cells was confirmed by western blot analysis of the expression of p53 and p53-target gene MDM2 (Fig. 1A). H1299 cells did not express p53 and MDM2 regardless of whether the cells were incubated with curcumin. By contrast, p53 as well as MDM2, were detected in A549 cells, and their expression was induced by curcumin treatment. These results confirmed the absence of p53 in H1299 cells, which were used in the subsequent experiments. The MTT assay showed that curcumin treatment time-dependently decreased the viability of the two cell lines $(\mathrm{P}<0.01)$ (Fig. 1B). Of note, the viability of A549 cells was affected by curcumin to a greater extant than that of H1299 cells; for instance, at the 16-h time-point, the proliferation rates of A549 and H1299 cells were 54 and $75 \%$, respectively. To further determine the mode of H1299 cell death, flow cytometric analysis was performed following double staining with Annexin V-FITC and PI. As shown in Fig. 1C and D, a significant and time-dependent induction of H1299-cell necrosis was detected following $16-24 \mathrm{~h}$ of curcumin treatment $(\mathrm{P}<0.05)$. However, apoptosis was not significantly induced in H1299 cells by treatment with $10 \mu \mathrm{M}$ curcumin for 8-24 h $(\mathrm{P}>0.05)$.

Curcumin-induced cell death is mitochondria-dependent. To investigate the underlying mechanisms of curcumin-induced H1299-cell necrosis, Bax and cytochrome $c$ expression and distribution were examined. The results demonstrated that curcumin induced Bax translocation to mitochondria and cytochrome $c$ release from the mitochondria in A549 and H1299 cells (Fig. 2A). The relative expression of Bax and cytochrome $c$ in mitochondria and cytosol were then quantified by densitometric analysis (Fig. 2B and C), showing that $10 \mu \mathrm{M}$ curcumin significantly and time-dependently enhanced Bax translocation and cytochrome $c$ release in the two cell lines $(\mathrm{P}<0.01$ and $\mathrm{P}<0.05$, respectively), particularly at the time-point of $24 \mathrm{~h}$. These results suggested that mitochondria have important roles in curcumin-induced necrosis of A549 and H1299 cells. 


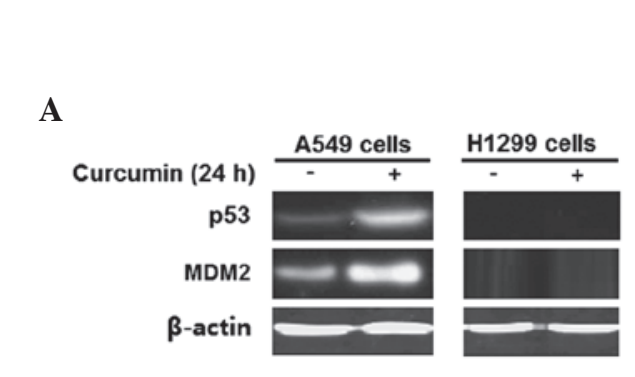

C
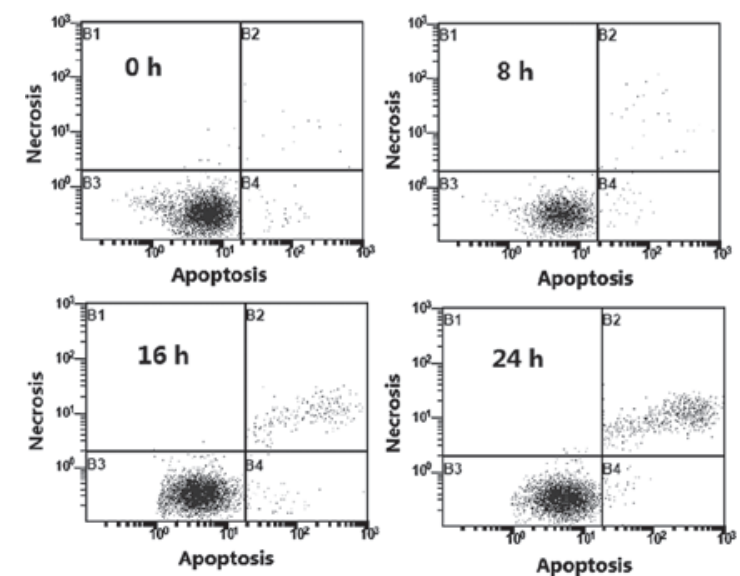

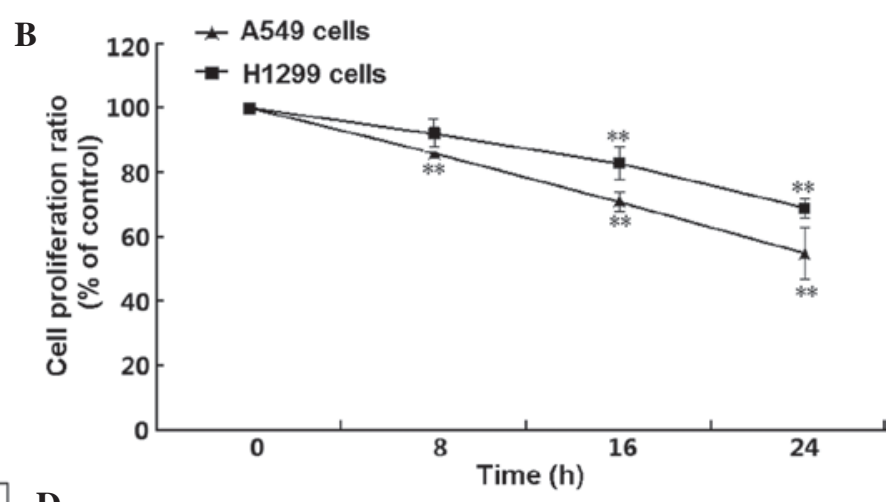

D

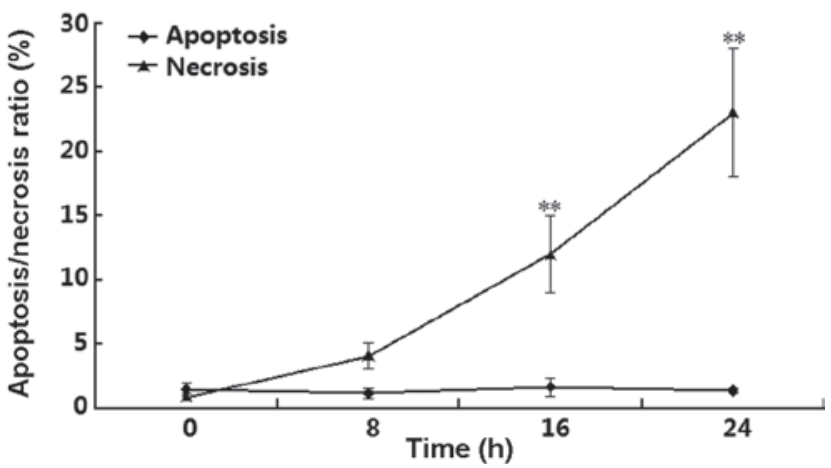

Figure 1. Curcumin induces necrosis of A549 and H1299 cells. Cells were incubated with $10 \mu \mathrm{M}$ curcumin for the indicated durations. (A) Examination of p53 status of H1299 cells using western blot analysis. (B) Curcumin reduced the viability of A549 and H1299 cells. (C) Flow cytometric analysis following propidium iodide (y-axis) and annexin V (x-axis) staining showed that curcumin induced necrotic cell death of H1299 cells. (D) Apoptotic and necrotic rates were determined by quantification of $\mathrm{C}$. Values are expressed as the mean \pm standard deviation. ${ }^{* *} \mathrm{P}<0.01$, compared to the control.

Caspase family members are activated in curcumin-treated $H 1299$ cells. To investigate the involvement of caspases in the programmed necrosis of H1299 cells, caspase activity was examined. As shown in Fig. 3A, a significant and time-dependent increase of caspase 3, 6 and 7 activity was observed following incubation with curcumin for 16-24 h $(\mathrm{P}<0.01)$. To confirm the involvement of caspases in the curcumin-induced death of H1299 cells, the effects of the classical caspase inhibitor z-VAD-FMK on H1299-cell necrosis were assessed using flow cytometry. As shown in Fig. 3B and C, after treatment with curcumin for $24 \mathrm{~h}$, the number of necrotic cells was significantly increased, which was almost completely abrogated in the presence of the caspase inhibitor. This result suggested that curcumin-induced necrosis in H1299 cells was caspase-dependent.

Oligomerization of Bax and Bak is involved in curcumin-induced H1299 cell necrosis. The present study further investigated whether oligomerization between Bax and Bak is involved in curcumin-induced H1299-cell necrosis. For this, mitochondrial lysates were reacted with a chemical cross-linker to stabilize the oligomers for western blot analysis. As shown in Fig. 4A, almost no Bax oligomer was induced during $0-8 \mathrm{~h}$ of curcumin treatment; however, after incubation for 16-24 h, curcumin induced an increase of mitochondrial Bax levels and Bax oligomerization. Furthermore, although no change in mitochondrial Bak levels was detected, Bak oligomerization was also induced. To further elucidate effects of this sub-cellular distribution of Bax and Bak in curcumin-induced
H1299 cell necrosis, the present study examined the cell death mode of wild-type (wt) H1299 cells as well as H1299 (Bax-/-) and H1299 (Bak-/-) cells, in which Bax and Bak had been silenced with specific siRNAs. The Bax and Bak knockdown efficiency was confirmed by western blot analysis (Fig. 4B). Following treatment with curcumin, a significant increase in the number of necrotic cells was observed in the H1299 (wt), H1299 (Bax-/-) and H1299 (Bak-/-) cells ( $\mathrm{P}<0.01)$; of note, the amount of necrotic H1299 cells (wt) was significantly greater than that in the Bax- or Bak knockdown H1299 cell groups (Fig. 4C). In addition, these effects were confirmed by caspase activation analysis, revealing a significant increase of caspase activity in all of the three H1299-cell groups $(\mathrm{P}<0.01)$. In parallel to the necrotic rates, curcumin-increased caspase activity in (Bax-/-) and in (Bak-/-) H1299 cells was lower than that in $\mathrm{H} 1299$ cells $(\mathrm{wt})(\mathrm{P}<0.01)$. All of these results suggested that Bax and Bak as well as their oligomerization are involved in the curcumin-induced necrosis of H1299 cells.

Inhibition of Bax and Bak attenuates cytochrome c release in H1299 cells. Cytochrome $c$ release levels in the H1299 (wt), H1299 (Bax-/-) and H1299 (Bak-/-) cells were examined. Fig. 5A and $\mathrm{B}$ shows that in the absence of curcumin, cytochrome $c$ was mainly localized to the mitochondria irrespective of the cells' Bax or Bak status. After treatment with curcumin, cytochrome $c$ release was significantly increased in the three groups $(\mathrm{P}<0.01)$; however, the release in the H1299 (Bax-/-) and (Bak-/-) H1299 cells was obviously lower than that in the H1299 (wt) cells $(\mathrm{P}<0.01)$. Total cytochrome $c$ expression levels in the three 


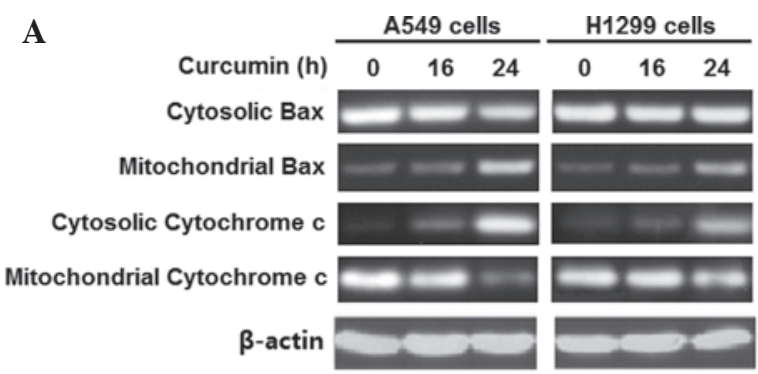

B

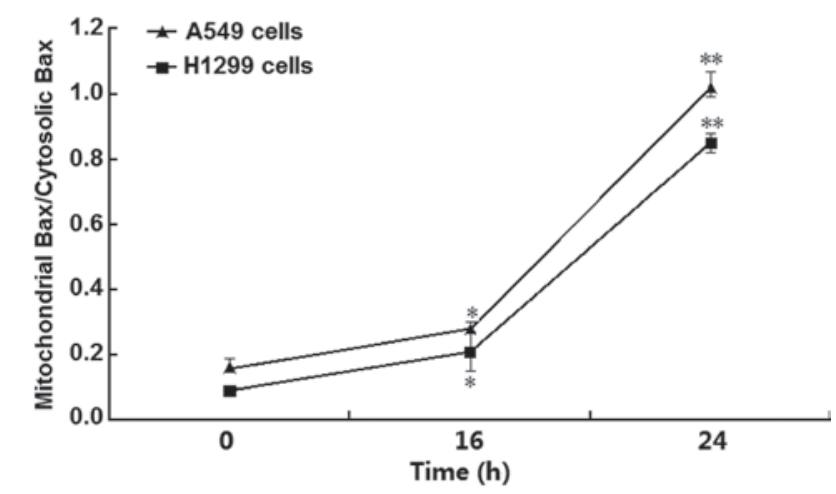

C

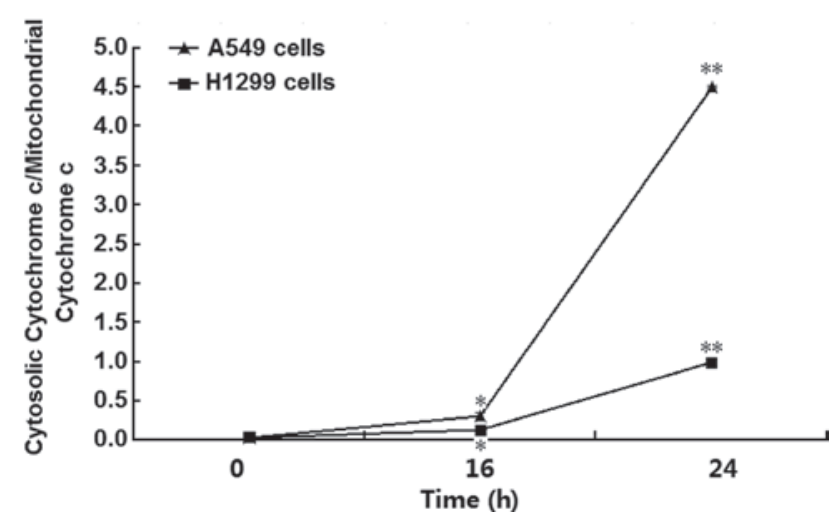

Figure 2. Curcumin induces changes in the Bax and cytochrome $c$ distribution in A549 and H1299 cells. (A) Western blot analysis of Bax and cytochrome cin A549 and H1299 cells following treatment with $10 \mu \mathrm{M}$ curcumin for 0,16 or $24 \mathrm{~h}$. Relative protein levels of (B) Bax and (C) cytochrome $c$ in mitochondria and cytosol. Values are expressed as the mean \pm standard deviation. ${ }^{*} \mathrm{P}<0.05$; ${ }^{* * *} \mathrm{P}<0.01$, compared to the control. Bax, B-cell lymphoma-2-associated $\mathrm{X}$ protein.

cell lines were also detected by western blot analysis, revealing that cytochrome $c$ expression levels in Bax- or Bak-deficient H1299 cells was lower than that in H1299 (wt) cells (Fig. 5C). However, no changes in the expression of mitochondrial CoxIV were observed in the three groups.

Overexpression of Bcl-2 attenuates necrosis and cytochrome c release in curcumin-treated $H 1299$ cells. Finally, the present study examined the effects of plasmid-mediated Bcl-2 overexpression on the toxicity of curcumin on H1299 cells. Transfection efficiency was confirmed by western blot analysis, which demonstrated that the Bcl-2 protein levels in the transfected H1299 cells were obviously higher than those in the untransfected H1299 cells (Fig. 6A). The transfected H1299 cells were then treated with curcumin and the cell

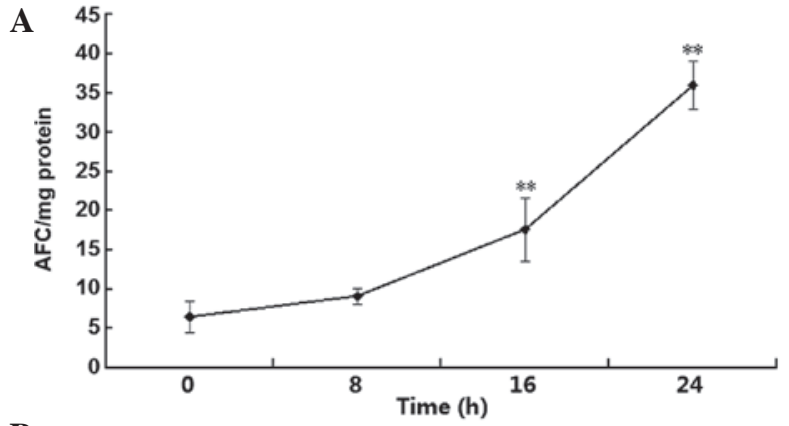

B
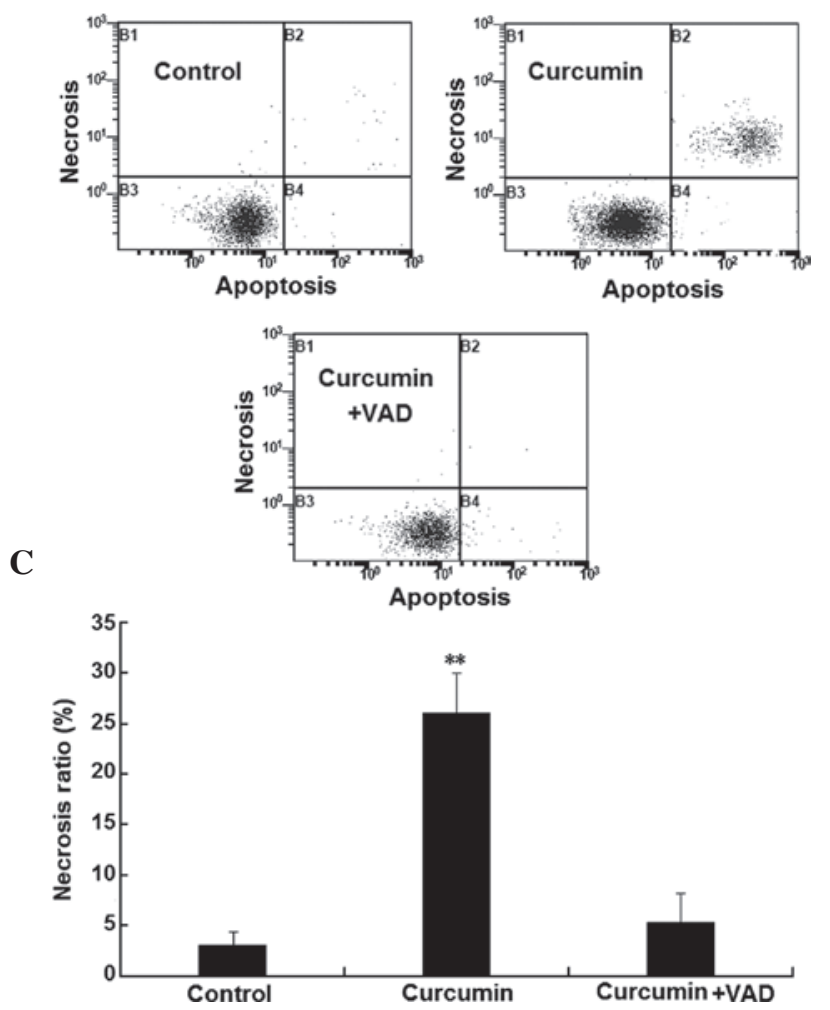

Figure 3. Curcumin increases caspase activity in H1299 cells (A) Cells were treated with $10 \mu \mathrm{M}$ curcumin for $16-24 \mathrm{~h}$ and caspase activity was detected fluorometrically. (B and C) Flow cytometric analysis showed that $100 \mu \mathrm{M}$ VAD inhibited the curcumin-induced necrosis of H1299 cells. Values are expressed as the mean \pm standard deviation. ${ }^{* *} \mathrm{P}<0.01$, compared to control. AFC, 7-amino-4-trifluoromethyl coumarin; VAD, Val-Ala-Asp.

viability was assessed using an MTT assay. As shown in Fig. 6B, curcumin significantly decreased the cell viability in wt as well as in Bcl-2-overexpressing $\mathrm{H} 1299$ cells $(\mathrm{P}<0.01)$. However, the cell death rate in the $\mathrm{Bcl}-2$ overexpressing cells was obviously lower than that in the wild-type H1299 cells $(\mathrm{P}<0.05)$. The effects of Bcl-2 on the cell viability were further proved by flow cytometry using double staining with Annexin V-FITC and PI. As shown in Fig. 6C, compared with the controls, the necrotic rate of wt H1299 cells was $22 \%$, which was reduced to $13 \%$ in the $\mathrm{Bcl}-2$ overexpressing H1299 cells. These results suggested that Bcl-2 attenuated curcumin-induced necrosis in H1299 cells.

\section{Discussion}

Curcumin, an active component of turmeric, which is used as a dietary supplement and a herbal medicine in numerous Asian 
A
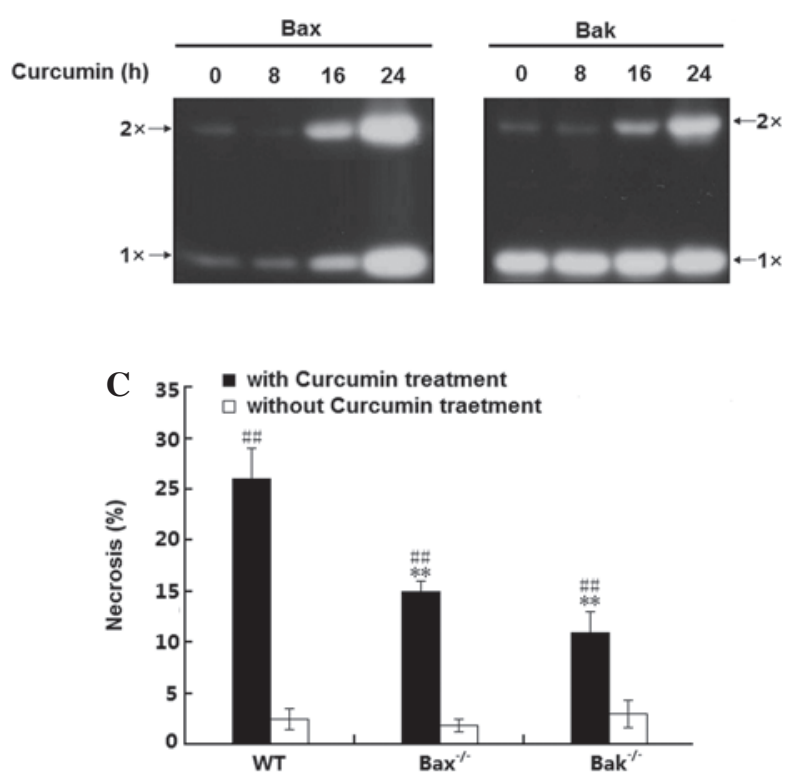
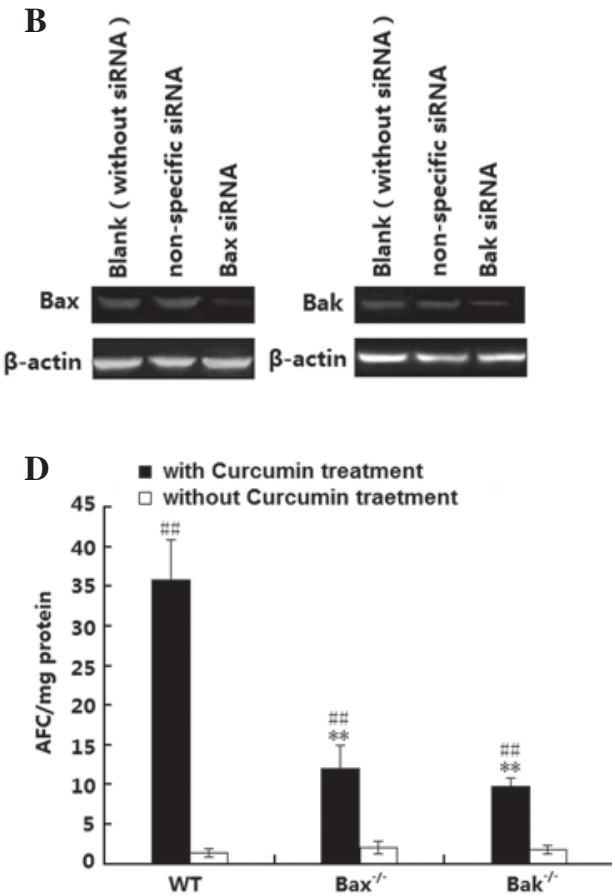

Figure 4. (A) Curcumin $(10 \mu \mathrm{M})$ induces Bax and Bak oligomerization in H1299 cells (wt) after treatment for 16-24 h. (B) Examination of Bax or Bak levels in H1299 cells (Bax-/-), and H1299 cells (Bak-/-). (C) Curcumin induced necrosis of the three indicated cell types following 24 h of incubation. (D) Curcumin increased caspase activity of the three indicated cell types at the $24 \mathrm{~h}$ time-point. Values are expressed as the mean \pm standard deviation. ${ }^{\#} \mathrm{P}<0.01$, compared to the control (without curcumin treatment); ${ }^{* *} \mathrm{P}<0.01$, compared to wt H1299 cells. wt, wild-type; AFC, 7-amino-4-trifluoromethyl coumarin; Bax, B-cell lymphoma-2-associated X protein; Bak, B-cell lymphoma-2 homologous antagonist killer; siRNA, small interfering RNA.

countries, has been demonstrated to have potent anti-neoplastic effects on a variety of cancer cell types in vitro as well as chemo-preventive effects in murine carcinoma models (5). Curcumin inhibits the proliferation and induces apoptosis of various types of cancer cell $(24,25)$. In agreement with previous studies, the present study revealed that after treatment for 16-24 h, $10 \mu \mathrm{M}$ curcumin significantly decreased the viability of p53-proficient A549 cells and p53-deficient H1299 cells. In addition, the mode of cell death of H1299 cells was analyzed using flow cytometry, which demonstrated that the curcumin treatment resulted in necrosis, while only few apoptotic cells were present. Furthermore, the present study revealed that mitochondria were involved in the curcumin-induced H1299-cell necrosis. In addition, Bax and Bak were activated, which resulted in cytochrome $c$ release caspase activation. Finally, the present study showed that Bax/Bak inhibition and Bcl-2 overexpression attenuated curcumin-induced H1299 cell necrosis. The present study indicated that the p53-independent mechanism of curcumin-induced necrosis of H1299 cells is associated with the mitochondria.

Apoptosis, an extensively studied and well-known type of $\mathrm{PCD}$, is a highly regulated process in all multicellular organisms and is essential for embryonic development and adult tissue homeostasis. It has been recognized that deregulation of apoptosis contributes to the development of cancers (6). Conventional chemotherapeutic agents were developed based on the fact that the proliferation of tumor cells is upregulated. By targeting intracellular molecules including the DNA or microtubules, chemotherapeutics induce various degrees of damage or stress in rapidly dividing cells, leading to apoptosis (26). p53 has important roles in apoptosis-inducing cancer therapies through initiating the DNA damage response $(27,28)$. p53 regulates DNA damage-induced apoptosis through a transcription-dependent pathway [involving MDM2 and p53-upregulated modulator of apoptosis (PUMA)], and/or a transcription-independent pathway (involving Bax, Bak and Bcl-2) (29,30). Of note, in the p53-deficient H1299 cells, Bax and Bak can be activated and form oligomers at mitochondrial sites, which may be involved in the regulation of the permeability of the mitochondrial outer membrane to induce permeability transition and cytochrome $c$ release, which can in turn activate the caspase cascade (31). Results of previous studies and the present study indicated that curcumin exerts its cytotoxic effects via p53-dependent as well as p53-independent mitochondria-associated cell death mechanisms.

Bcl-2 family members are key regulators of mitochondria-dependent cell death. The molecular basis of the regulation of apoptosis through either pro-apoptotic members (Bax/Bak) or anti-apoptotic Bcl-2 is well known. Bcl-2 family proteins regulate mitochondrial apoptosis or/and necrosis through controlling the permeability of the mitochondrial membrane (32). However, with regard to the mechanism of action of curcumin on the p53-deficient H1299 cell line, it remains elusive how cell death signaling is initiated and how Bax and Bak are upregulated. Several potential pathways of p53-independent apoptosis have been previously reported. For instance, in doxorubicin-treated Saos-2 cells, overexpression of p73 $\gamma$, a p53 family member, caused apoptosis and PUMA transactivation, which in turn activated mitochondrial translocation of Bax and cytochrome $c$ release (33). Another study reported that in p53-mutant HT-29 colon cancer cells, quercetin induced apoptosis by decreasing the mitochondrial 


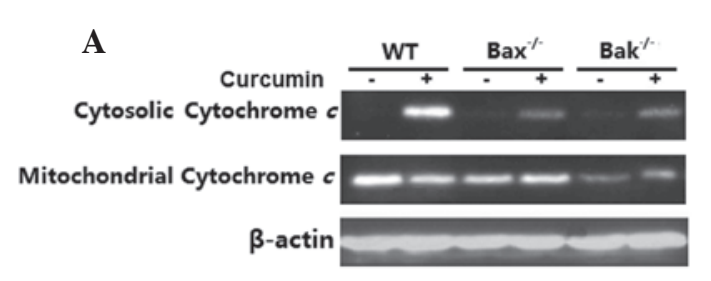

B

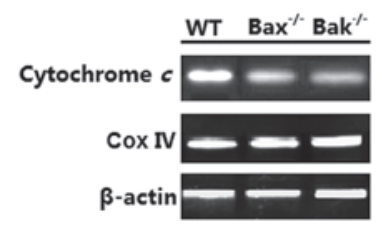

C

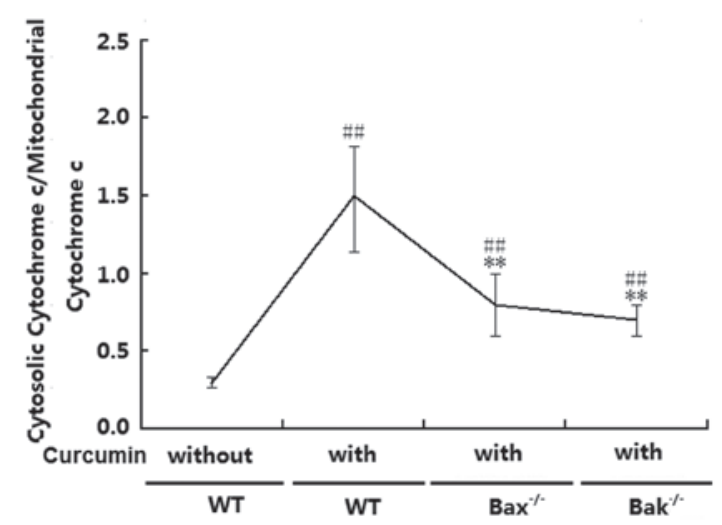

Figure 5. Deficiency of Bax or Bak attenuates the release of cytochrome $c$ in H1299 cells treated with $10 \mu \mathrm{M}$ curcumin for $24 \mathrm{~h}$. (A) Western blot analysis of the cytosolic and mitochondrial levels of cytochrome $c$. (B) Cytochrome $c$ release levels in the cytosol and mitochondria. (C) Total cytochrome $c$ and CoxIV expression levels in whole-cell lysates of wt, Bax (-/-) and Bak (-/-) $\mathrm{H} 1299$ cells. Values are expressed as the mean \pm standard deviation. ${ }^{\# \#} \mathrm{P}<0.01$, compared to the control; ${ }^{* *} \mathrm{P}<0.01$, compared to wt H1299 cells. Bax, B-cell lymphoma-2-associated X protein; Bak, B-cell lymphoma-2 homologous antagonist killer; Cox, cyclooxygenase; wt, wild-type

membrane potential, and increasing reactive oxygen species production and sestrin 2 expression through the adenosine monophosphate-activated protein kinase/p38 pathway (34). These previous studies, combined with the results of the present study, indicated that p53-dependent and p53-independent regulatory mechanisms may simultaneously contribute to the activation of Bax/Bak, which in turn trigger apoptosis; however, further studies are required to determine the exact underlying molecular mechanism.

Another noteworthy finding of the present study was the inter-dependence between Bax and Bak with regard to their activation in curcumin-treated H1299 cells. Bax and Bak were activated in $\mathrm{H} 1299$ cells (wt), while Bax and Bak translocation and oligomerization was partially inhibited in H1299 (Bak-/-) and H1299 (Bax-/-) cells. Furthermore, the anti-apoptotic protein $\mathrm{Bcl}-2$ was shown to suppress curcumin-induced H1299 cell necrosis through abrogating the Bax - Bak interaction and inhibiting cytochrome $c$ release. It remains elusive whether this Bax - Bak inter-dependence is linked to their function of permeabilizing the outer mitochondrial membrane. Previous studies suggested several possibilities; for instance, Bax and Bak contain a hydrophobic BH3 domain, which only becomes available for binding
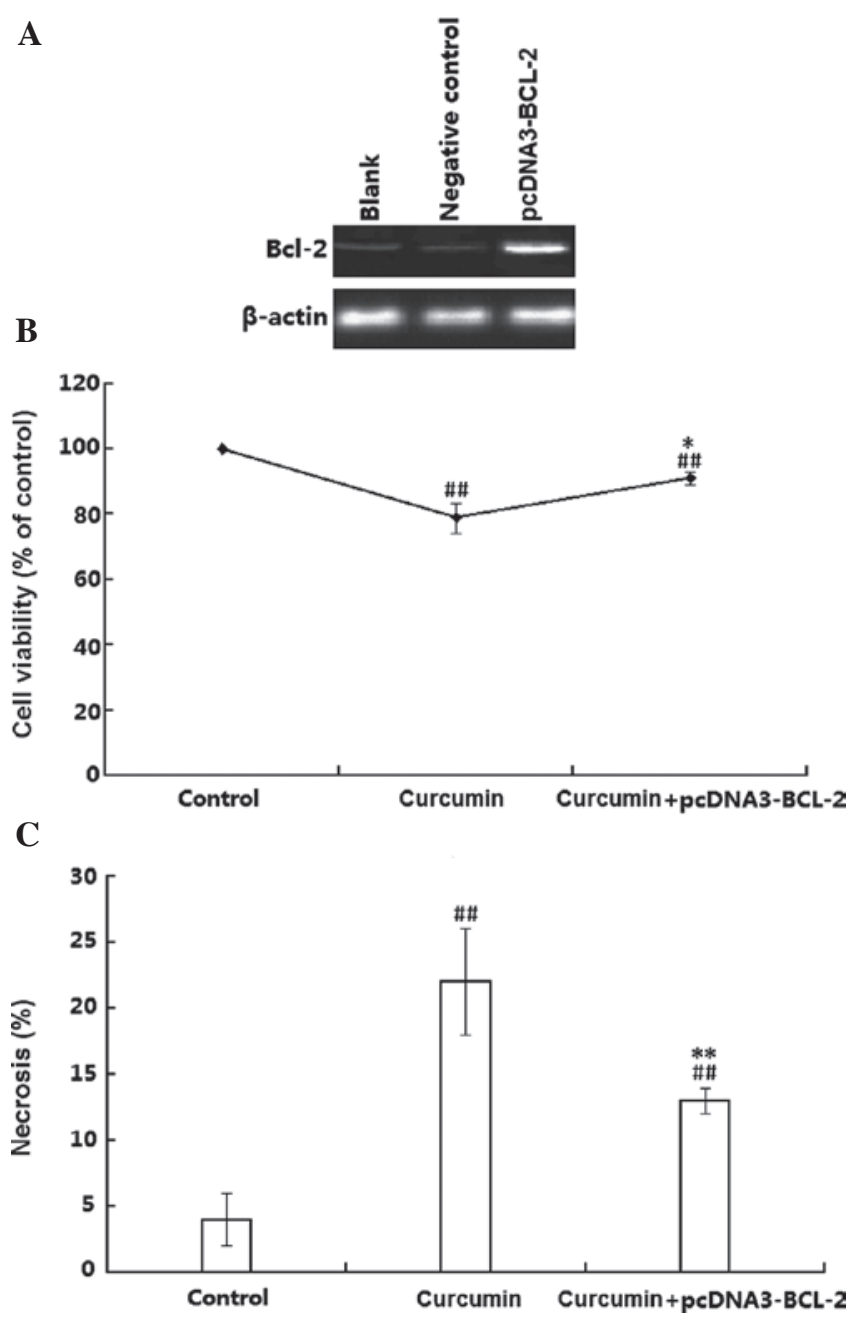

Figure 6. (A) Western blot analysis of Bcl-2 expression in H1299 cells after transfection with Bcl-2 overexpression vector. (B) Overexpression of Bcl-2 attenuated curcumin-decreases in H1299 cell viability. (C) Overexpression of Bcl-2 attenuated curcumin-induced $\mathrm{H} 1299$ cell necrosis. Values are expressed as the mean \pm standard deviation. ${ }^{\# \#} \mathrm{P}<0.01$, compared to the control; ${ }^{*} \mathrm{P}<0.05$ and ${ }^{* * *} \mathrm{P}<0.01$, compared to wild-type H1299 cells. Bcl-2, B-cell lymphoma 2.

during activation to facilitate hetero- and homo-oligomerization $(35,36)$. Furthermore, TNF- $\alpha$-induced mitochondrial cytochrome $c$ release and apoptosis of HeLa cells was associated with simultaneous homo-oligomerization and hetero-oligomerization of Bax/Bak (37). The present study indicated that Bax and Bak may function cooperatively and interdependently to permeabilize the outer mitochondrial membrane, resulting in the release of cytochrome $c$ and activation of the caspase cascade.

Two pathways have been confirmed to be involved in apoptosis induction, including death receptor-dependent extrinsic and mitochondria-dependent intrinsic pathways $(17,38)$. The intrinsic apoptotic pathway mediated by mitochondria is mainly triggered by the collapse of the mitochondrial membrane potential (39). The collapse prompts the release of pro-apoptotic cytochrome $c$ into the cytoplasm, which has been proposed as a 'point of no return' in the mitochondrial pathway $(40,41)$. Once mitochondria sense the cell death stimuli, cytochrome $c$ is released into the cytosol, where it binds to Apaf- 1 and caspase- 9 to form a large complex termed the apoptosome, which in turn initiates the activation of 
downstream caspases 3, -6 and -7 , as well as other apoptotic effectors; therefore, activation of the caspase-cascade system is the key event of mitochondria-dependent apoptosis $(42,43)$. In addition, several previous studies suggested that the execution of necrotic cell death is caspase-independent $(12,44)$. However, in the curcumin-treated H1299 cell model used in the present study, caspase activation was induced simultaneously with the appearance of cell necrosis. These results strongly indicated that apoptosis and necrosis may share common features, such as caspase activation.

In conclusion, the results of the present study revealed that curcumin exerts cytotoxic effects by inducing necrosis in the p53-deficient H1299 cell line through a mitochondria-associated pathway. The p53-independent mechanism was shown to include Bax/Bak translocation to mitochondria, where they permeabilized the outer mitochondrial membrane to trigger a cell death cascade. These results supported the potential of curcumin as a promising agent for the treatment of p53-deficient lung cancers.

\section{Acknowledgements}

The present study was supported by grants from the Science Technology Study of Hubei Department of Education Foundation (grant no. Q20141677).

\section{References}

1. O'Sullivan-Coyne G, O'Sullivan GC, O'Donovan TR, Piwocka K and McKenna SL: Curcumin induces apoptosis-independent death in oesophageal cancer cells. Br J Cancer 101: 1585-1595, 2009.

2. Friedman L, Lin L, Ball S, Bekaii-Saab T, Fuchs J, Li PK, Li C and Lin J: Curcumin analogues exhibit enhanced growth suppressive activity in human pancreatic cancer cells. Anticancer Drugs 20: 444-449, 2009.

3. Steward WP and Gescher AJ: Curcumin in cancer management: Recent results of analogue design and clinical studies and desirable future research. Mol Nutr Food Res 52: 1005-1009, 2008.

4. Shehzad A, Lee J, Huh TL and Lee YS: Curcumin induces apoptosis in human colorectal carcinoma (HCT-15) cells by regulating expression of Prp4 and p53. Mol Cells 35: 526-532, 2013.

5. Choudhuri T, Pal S, Agwarwal ML, Das T and Sa G: Curcumin induces apoptosis in human breast cancer cells through p53-dependent Bax induction. FEBS Lett 512: 334-340, 2002.

6. Alenzi FQ, Wyse RK and Altamimi WG: Apoptosis as a tool for therapeutic agents in haematological diseases. Expert Opin Biol Ther 4: 407-420, 2004.

7. Huang S, Shao K, Liu Y, Kuang Y, Li J, An S, Guo Y, Ma H and Jiang C: Tumor-targeting and microenvironment-responsive smart nanoparticles for combination therapy of antiangiogenesis and apoptosis. ACS Nano 7: 2860-2871, 2013.

8. Griffith TS: Induction of tumor cell apoptosis by TRAIL gene therapy. Methods Mol Biol 542: 315-334, 2009.

9. Sosna J, Voigt S, Mathieu S, Lange A, Thon L, Davarnia P, Herdegen T, Linkermann A, Rittger A, Chan FK, et al: TNF-induced necroptosis and PARP-1-mediated necrosis represent distinct routes to programmed necrotic cell death. Cell Mol Life Sci 71: 331-348, 2014.

10. Proskuryakov SY, Gabai VL and Konoplyannikov AG: Necrosis is an active and controlled form of programmed cell death. Biochemistry (Mosc) 67: 387-408, 2002.

11. Vandenabeele, P., W. Declercq, F. Van Herreweghe, et al. The role of the kinases RIP1 and RIP3 in TNF-induced necrosis. Sci Signal. 2010; 115: re4.

12. Baritaud M, Boujrad H, Lorenzo HK, Krantic S and Susin SA Histone H2AX: The missing link in AIF-mediated caspase-independent programmed necrosis. Cell Cycle 9: 3166-3173, 2010.
13. Iwasaki R, Ito K, Ishida T, Hamanoue M, Adachi S, Watanabe T and Sato Y: Catechin, green tea component, causes caspase-independent necrosis-like cell death in chronic myelogenous leukemia. Cancer Sci 100: 349-356, 2009.

14. Nicotera P and Melino G: Regulation of the apoptosis-necrosis switch. Oncogene 23: 2757-2765, 2004.

15. Lin X, Sun T, Cai M and Shen P: Cell-death-mode switch from necrosis to apoptosis in hydrogen peroxide treated macrophages. Sci China Life Sci 53: 1196-1203, 2010.

16. Bergeron L, Perez GI, Macdonald G, et al: Defects in regulation of apoptosis in caspase-2-deficient mice. Genes Dev 12: 1304-1314, 1998.

17. Hosseinimehr SJ, Azadbakht M, Tanha M, Mahmodzadeh A and Mohammadifar S: Protective effect of hawthorn extract against genotoxicity induced by methyl methanesulfonate in human lymphocytes. Toxicol Ind Health 27: 363-369, 2010.

18. Kosmider B, Wojcik I, Osiecka R, Bartkowiak J, Zyner E, Ochocki J and Liberski P: Enhanced P53 and BAX gene expression and apoptosis in A549 cells by cis-Pt(II) complex of 3-aminoflavone in comparison with cis-DDP. Invest New Drugs 23: 287-297, 2005.

19. Song G, Mao YB, Cai QF, Yao LM, Ouyang GL and Bao SD: Curcumin induces human HT-29 colon adenocarcinoma cell apoptosis by activating p53 and regulating apoptosis-related protein expression. Braz J Med Biol Res 38: 1791-1798, 2005.

20. Yu JL, Rak JW, Coomber BL, et al: Effect of p53 status on tumor response to antiangiogenic therapy. Science 295:1526-1528, 2002.

21. Tian F, Song M, Xu PR, et al: [Curcumin promotes apoptosis of esophageal squamous carcinoma cell lines through inhibition of NF-kappaB signaling pathway]. Ai Zheng 27: 566-570, 2008 (In Chinese)

22. Zhang W, Liu N, Wang X, et al: Benzo(a)pyrene-7,8-diol9,10-epoxide induced p53-independent necrosis via the mitochondria-associated pathway involving Bax and Bak activation. Hum Exp Toxicol 34: 179-190, 2015.

23. Guo BC and Xu YH: Bcl-2 over-expression and activation of protein kinase $C$ suppress the trail-induced apoptosis in Jurkat $\mathrm{T}$ cells. Cell Res 11: 101-106, 2001.

24. Bar-Sela G, Epelbaum R and Schaffer M: Curcumin as an anti-cancer agent: Review of the gap between basic and clinical applications. Curr Med Chem 17: 190-197, 2010.

25. Tian C, Xing G, Xie P, et al: KRAB-type zinc-finger protein Apak specifically regulates p53-dependent apoptosis. Nat Cell Biol 11: 580-591, 2009.

26. Blankenberg FG: Apoptosis imaging: Anti-cancer agents in medicinal chemistry. Anticancer Agents Med Chem 9: 944-951, 2009.

27. Meulmeester E and Jochemsen AG: p53: A guide to apoptosis. Curr Cancer Drug Targets 8: 87-97, 2008.

28. Xu Y: Regulation of $\mathrm{p} 53$ responses by post-translational modifications. Cell Death Differ 10: 400-403, 2003.

29. Leibowitz BJ, Qiu W, Liu H, Cheng T, Zhang L and Yu J: Uncoupling $\mathrm{p} 53$ functions in radiation-induced intestinal damage via PUMA and p21. Mol Cancer Res 9: 616-625, 2011.

30. Thakur VS, Ruhul Amin AR, Paul RK, Gupta K, Hastak K, Agarwal MK, Jackson MW, Wald DN, Mukhtar H and Agarwal ML: p53-Dependent p21-mediated growth arrest pre-empts and protects HCT116 cells from PUMA-mediated apoptosis induced by EGCG. Cancer Lett 296: 225-232, 2010.

31. Morales-Cruz M,Figueroa CM, Gonzalez-Robles T, et al: Activation of caspase-dependent apoptosis by intracellular delivery of cytochrome c-based nanoparticles. J Nanobiotechnology 12: 33, 2014.

32. Antignani A and Youle RJ: How do Bax and Bak lead to permeabilization of the outer mitochondrial membrane? Curr Opin Cell Biol 18: 685-689, 2006.

33. Holcakova J, Ceskova P, Hrstka R, Muller P, Dubska L, Coates PJ, Palecek E and Vojtesek B: The cell type-specific effect of TAp73 isoforms on the cell cycle and apoptosis. Cell Mol Biol Lett 13: 404-420, 2008

34. Kim GT, Lee SH, Kim JI and Kim YM: Quercetin regulates the sestrin 2-AMPK-p38 MAPK signaling pathway and induces apoptosis by increasing the generation of intracellular ROS in a p53-independent manner. Int J Mol Med 33: 863-869, 2014.

35. Kim H, Tu HC, Ren D, et al: Stepwise activation of BAX and BAK by tBID, BIM, and PUMA initiates mitochondrial apoptosis. Mol Cell 36: 487-499, 2009.

36. Antonsson B, Montessuit S, Lauper S, et al: Bax oligomerization is required for channel-forming activity in liposomes and to trigger cytochrome c release from mitochondria. Biochem J 345 (Pt 2): 271-278, 2000. 
37. Sundararajan R, Cuconati A, Nelson D and White E: Tumor necrosis factor-alpha induces Bax-Bak interaction and apoptosis, which is inhibited by adenovirus E1B 19K. J Biol Chem 276 45120-45127, 2001.

38. Wachter F, Grunert M, Blaj C, Weinstock DM, Jeremias I and Ehrhardt H: Impact of the p53 status of tumor cells on extrinsic and intrinsic apoptosis signaling. Cell Commun Signal 11: 27, 2013.

39. Mehmeti I, Gurgul-Convey E, Lenzen S and Lortz S: Induction of the intrinsic apoptosis pathway in insulin-secreting cells is dependent on oxidative damage of mitochondria but independent of caspase-12 activation. Biochim Biophys Acta 1813: 1827-1835, 2011.

40. Buratta M, Castigli E, Sciaccaluga M, Pellegrino RM, Spinozzi F, Roberti R and Corazzi L: Loss of cardiolipin in palmitate-treated GL15 glioblastoma cells favors cytochrome c release from mitochondria leading to apoptosis. J Neurochem 105: 1019-1031, 2008
41. Lee HJ, Lee HJ, Lee EO, Ko SG, Bae HS, Kim CH, Ahn KS, Lu J and Kim SH: Mitochondria-cytochrome C-caspase-9 cascade mediates isorhamnetin-induced apoptosis. Cancer Lett 270: 342-353, 2008

42. Nakabayashi J and Sasaki A: A mathematical model for apoptosome assembly: The optimal cytochrome c/Apaf-1 ratio. J Theor Biol 242: 280-287, 2006.

43. Adrain C and Martin SJ: The mitochondrial apoptosome: A killer unleashed by the cytochrome seas. Trends Biochem Sci 26: 390-397, 2001.

44. Kihana T, Tsuda H, Teshima S, et al: High incidence of p53 gene mutation in human ovarian cancer and its association with nuclear accumulation of $\mathrm{p} 53$ protein and tumor DNA aneuploidy. Jpn J Cancer Res 83: 978-984, 1992. 